\title{
Effects of airway infection by Pseudomonas aeruginosa: a computed tomographic study
}

\author{
Katherine A Miszkiel, Athol U Wells, Michael B Rubens, Peter J Cole, \\ David M Hansell
}

\begin{abstract}
Background - Pseudomonas aeruginosa commonly infects the airways of patients with bronchiectasis. A study was undertaken to examine the relationship between infection of the airways with this pathogen, the morphological pattern of bronchiectasis on thin section computed tomographic (CT) scanning, symptom duration, smoking habits of the patients, and the presence of airflow obstruction.

Methods - Thin section CT scans of 22 adult patients with bronchiectasis and concurrent sputum infected by $P$ aeruginosa $(\mathrm{Pa}+\mathrm{ve})$ and those of 45 randomly selected patients not infected by $P$ aeruginosa (Pa - ve) were analysed independently by two thoracic radiologists. Patients with cystic fibrosis were excluded. Each scan was scored at a lobar level for extent of bronchiectasis, severity of bronchial wall thickening and dilatation, predominant pattern of bronchiectasis, presence of mucus plugging, and degree of decreased attenuation of the lung parenchyma.

Results - The Pa + ve group had more extensive bronchiectasis and a greater degree of bronchial wall thickening and dilatation on the CT scan than the Pa - ve group; more extensive decreased attenuation was seen in the $\mathbf{P a}+$ ve group. These findings were robust on multivariate analysis; decreased attenuation was also independently related to the duration of sputum production.

Conclusion - Patients with bronchiectasis infected by $P$ aeruginosa have more extensive and severe bronchiectasis on thin section CT scanning than those without $P$ aeruginosa infection. The bronchi and small airways are both involved, reflecting the end result of complex interactions between host airways and the numerous virulence factors produced by $P$ aeruginosa. (Thorax 1997;52:260-264)
\end{abstract}

Keywords: Pseudomonas aeruginosa, bronchiectasis, thin section CT scanning.

Infection of the airways by Pseudomonas aeruginosa occurs commonly in patients with cystic fibrosis ${ }^{1}$ but also occurs in patients with other forms of bronchiectasis. ${ }^{2}$ Although much of the morbidity and mortality of patients with bronchiectasis may be due to chronic infection with $P$ aeruginosa, chronic infection of the airways with this pathogen in patients with cystic fibrosis can be prevented by early institution of anti-pseudomonas chemotherapy. ${ }^{3}$ Experiments in vitro have suggested that $P$ aeruginosa plays an important part in the progression of bronchiectasis, ${ }^{4-6}$ and mucociliary clearance measurements ${ }^{7}$ and results in an experimental model of bronchiectasis in vitro are consistent with this. ${ }^{8}$ Computed tomographic (CT) scanning is an accurate and non-invasive method of imaging the bronchial tree and is the technique of choice for the diagnosis of bronchiectasis. ${ }^{9-11}$

Nagaki et al investigated a group of nonsmoking patients with a clinical diagnosis of chronic bronchitis whose airways had been infected by $P$ aeruginosa for at least one year. They found that such patients had significantly more severe bronchiectasis on CT scanning than non-infected matched patients and asymptomatic controls. ${ }^{12}$ Similar results were obtained in a study performed at our institution in which patients infected with $P$ aeruginosa appeared to have more extensive bronchiectasis on CT scanning and more severe airflow obstruction than non-infected patients, independent of their smoking history. ${ }^{13}$ However, in both these studies the assessment of bronchiectasis on the CT scan was limited.

In this study we have examined in more detail the relationship between infection of the airways by $P$ aeruginosa and the morphological pattern of bronchiectasis on thin section CT scans, and any relation between these and the severity and duration of symptoms, patients' smoking habits, and respiratory function.

\section{Methods}

Consecutive adult patients undergoing investigation for known or suspected bronchiectasis between January 1991 and March 1993 were studied, provided that bronchiectasis was present on thin section CT scanning and either mucoid or non-mucoid strains of $P$ aeruginosa were cultured from sputum within three months of the scan. Exclusion criteria were (1) an abnormal sweat sodium concentration (tested in all patients), (2) a history of lobectomy or pneumonectomy (since this would affect the forced expiratory volume in one second $\left(\mathrm{FEV}_{1}\right)$ and alter the CT scores), and (3) age below 16 years. The control group consisted of randomly selected patients with chronic purulent sputum production and bronchiectasis on CT scanning who were not infected by $P$ aeruginosa. Twenty two patients infected with $P$ aeruginosa and 45 controls were studied. The following information was ex- 
Table 1 Comparison of the demographic and clinical features obtained from the case records in patients with sputum infected with Pseudomonas aeruginosa ( $\mathrm{Pa}+$ ve group) and those not infected ( $\mathrm{Pa}-\mathrm{ve})$.

\begin{tabular}{lllr}
\hline & $\begin{array}{c}P a+\text { ve group } \\
(n=22)\end{array}$ & $\begin{array}{l}P a-\text { ve group } \\
(n=45)\end{array}$ & $p$ value \\
\hline Age (years) & $54.1(14.5)$ & $43.5(14.3)$ & $<0.01$ \\
Sex (M:F) & $10: 12$ & $16: 29$ & 0.44 \\
No. of smokers (ever vs never) & 9 & 16 & 0.67 \\
Median (range) history (pack years) & $0(0-40)$ & 18 & 0.46 \\
No. with history of asthma & 12 & $21.2(18.3)$ & 0.26 \\
Age at onset of sputum production (years) & $27.0(22.0)$ & 0.26 \\
Duration of sputum production (years) & $27.1(14.6)$ & $22.3(17.4)$ & 0.27 \\
FEV $_{1}$ (\% pred) & $58.2(30.1)$ & $75.4(22.9)$ & $<0.02$ \\
\hline
\end{tabular}

$\mathrm{FEV}_{1}=$ forced expiratory volume in one second. Values are mean (SD) on median (range).

tracted from case records: (1) demographic details; (2) smoking history ("ever" versus "never", pack years); (3) clinical history of asthma; (4) age at onset of sputum production; (5) duration of sputum production; (6) a predisposing cause, if any, for bronchiectasis. The percentage predicted $\mathrm{FEV}_{1}$ was also recorded. ${ }^{14}$

The CT scans were performed on an ultrafast electron beam scanner (Imatron C-100, San Francisco, USA) with a scan time of $200 \mathrm{~ms}$. Patients were scanned in full suspended inspiration from lung apex to diaphragm and $3 \mathrm{~mm}$ sections were obtained at $10 \mathrm{~mm}$ intervals. A high spatial resolution reconstruction algorithm was used and images were photographed on appropriate lung window settings (level $-700 \mathrm{HU}$, width $1500 \mathrm{HU}$ ). Two thoracic radiologists (DMH and MBR) independently analysed the CT scans in random order with no knowledge of which patients were infected with $P$ aeruginosa.

All CT observations were made at a lobar level with the lingula considered a separate lobe. The presence or absence of bronchiectasis
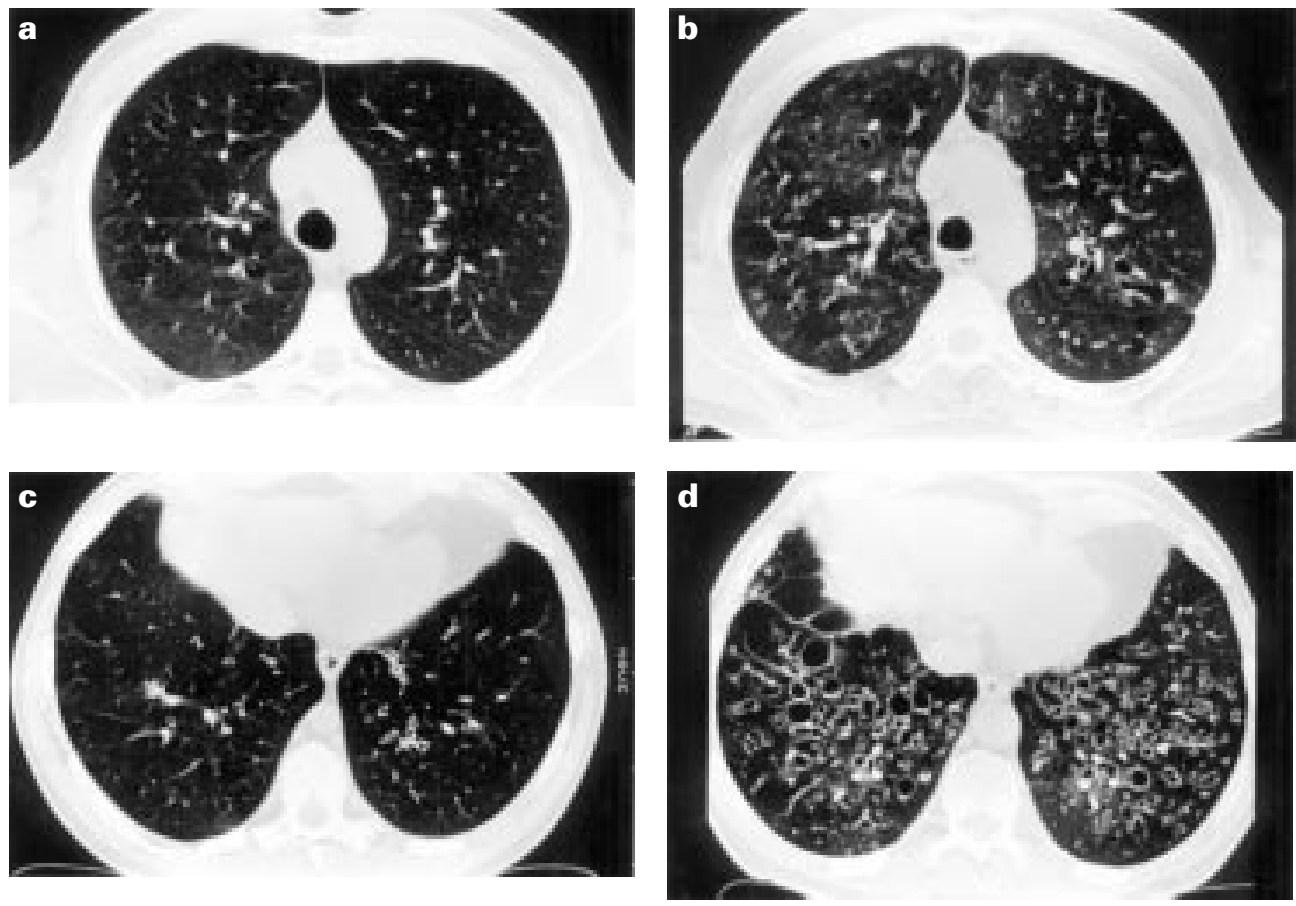

on the CT scans was determined according to the criteria described by Naidich. ${ }^{15}$ The CT scoring system used was that described by Reiff et al which has good interobserver agreement. ${ }^{16}$ The extent of bronchiectasis, severity of bronchial wall thickening, and severity of bronchial wall dilatation were recorded for each lobe using a four point scoring system (0-3): grade $0=$ no bronchiectasis, normal bronchial wall thickness, or no bronchial wall dilatation; grade $1=$ bronchiectasis present in none or one bronchopulmonary segment, bronchial wall thickening equivalent to half the diameter of the adjacent vessel, or bronchial wall dilatation equivalent to less than twice the diameter of the adjacent vessel; grade $2=$ bronchiectasis present in more than one bronchopulmonary segment, bronchial wall thickening equivalent to $0.5-1 \times$ diameter of the adjacent vessel or bronchial wall dilatation equivalent to $2-3 \times$ diameter of the adjacent vessel; grade $3=$ gross cystic bronchiectasis present, bronchial wall thickening equivalent to more than the diameter of the adjacent vessel, or bronchial wall dilation present equivalent to more than three times the diameter of the adjacent vessel. The total score for each of these parameters was obtained by adding together the scores for the six lobes.

The predominant pattern of bronchiectasis present in each lobe as classified by Reid (cylindrical, varicose or cystic) was recorded. ${ }^{17}$ The presence of either small centrilobular plugs or large plugs (plugging of the segmental and subsegmental airways) was recorded as present or absent. To assess involvement of the bronchioles by the disease process ${ }^{18}$ areas of decreased attenuation of the lung parenchyma

Figure 1 Thin section slices of a CT scan taken at two similar levels fom two patients with bronchiectasis, both with a 29 year duration of sputum production. Sections (a) and (b) are through the upper zones and (c) and (d) are through the lower zones. Sections (a) and (c) are from a patient not infected with $P$ aeruginosa and (b) and (d) are from a patient infected with $P$ aeruginosa. 
Table 2 Median scores (with ranges) for morpholopical features on CT scans in patients with sputum infected with Pseudomonas aeruginosa $(\mathrm{Pa}+\mathrm{ve})$ and those not infected (Pa $-v e)$.

\begin{tabular}{llll}
\hline & $\begin{array}{l}P a+\text { ve group } \\
(n=22)\end{array}$ & $\begin{array}{l}P a-\text { ve group } \\
(n=45)\end{array}$ & p value \\
\hline Extent of bronchiectasis & $9.5(3.5-14.0)$ & $5.0(1.5-15.5)$ & $<0.0001$ \\
Bronchial wall dilatation & $9.5(2.5-17.5)$ & $4.0(1.0-17.5)$ & $<0.0005$ \\
Bronchial wall thickening & $6.0(2.5-12)$ & $3.5(0-17.5)$ & $<0.0001$ \\
Global decreased attenuation & $5.5(0-12)$ & $2.0(0-11)$ & $<0.0005$ \\
Large plug score & $0.5(0-2.5)$ & $0(0-2.5)$ & 0.07 \\
Centrilobular plug score & $0.5(0-5)$ & $0(0-5)$ & 0.33 \\
\hline
\end{tabular}

Statistical analysis performed by Wilcoxon's rank sum test.

were recorded as: grade $0=$ normal, grade $1=$ decreased attenuation involving $<50 \%$ of the lobe, grade $2=$ decreased attenuation involving $>50 \%$ of the lobe; the total decreased attenuation score was the sum of the lobar scores. The mean scores of the two observers were used in the analysis.

Results are expressed as medians with ranges. A p value of $<0.05$ was regarded as statistically significant. Group comparisons were made using the Student's $t$ test, $\chi^{2}$ statistics, or Wilcoxon's rank sum test. Agreement between observers was expressed as the kappa coefficient; kappa values of $>0.60$ were taken to indicate good agreement between observers. ${ }^{19}$ Correlations were examined by multiple linear regression or, when appropriate, by logistic regression (Stata Data Analysis, Computing Resonance Center, Santa Monica, California, USA).

\section{Results \\ During the study period 327 patients with chronic purulent sputum and known or sus- pected bronchiectasis underwent CT scanning. Thirty five $(10.7 \%)$ were infected with $P$ aeru- ginosa, but 13 were excluded because of pre- vious pneumonectomy or lobectomy $(\mathrm{n}=9), P$ aeruginosa not cultured within three months of CT scan $(\mathrm{n}=2)$, and incomplete data $(\mathrm{n}=2)$. Of the remaining 22 patients, $14(63.6 \%)$ were continuously infected by $P$ aeruginosa. Patients positive for $P$ aeruginosa and controls were well matched for sex, history of asthma, the age at onset of sputum production, and the duration of sputum production (table 1 ). Five of the 22 patients $(22.7 \%)$ infected with $P$ aeruginosa $(\mathrm{Pa}$}

Table 3 Multiple regression analysis of independent relationships between FEV morphological features in the CT scan (examined as dependent variables), duration of sputum production and presence or absence of Pseudomonas aeruginosa. Age was also included as a covariate.

\begin{tabular}{lll}
\hline & $\begin{array}{l}\text { Duration of } \\
\text { sputum production }\end{array}$ & $\begin{array}{l}\text { Presence/absence of } \\
\text { P aeruginosa }\end{array}$ \\
\hline Extent of bronchiectasis & $0.02(-0.03$ to 0.06$)$ & $\begin{array}{l}3.4(1.7 \text { to } 5.0) \\
\mathrm{p}<0.0005\end{array}$ \\
Bronchial wall dilation & $\mathrm{p}=0.50$ & $4.2(2.0$ to 6.5$)$ \\
Bronchial wall thickness & $0.04(-0.03$ to 0.10$)$ & $\mathrm{p}<0.0005$ \\
& $\mathrm{p}=0.43$ & $2.7(1.5$ to 3.8$)$ \\
Global decreased attenuation & $0.02(-0.01$ to 0.05$)$ & $\mathrm{p}<0.0005$ \\
Large plug score & $0.05(0.00$ to 0.09$)$ & $\mathrm{p}<0.005$ \\
Centrolobular plug score & $\mathrm{p}<0.05$ & $0.26(-0.14$ to 0.65$)$ \\
FEV & $0.00(-0.01$ to 0.02$)$ & $\mathrm{p}=0.26$ \\
& $\mathrm{p}=0.38$ & $0.34(-0.29$ to 0.97$)$ \\
& $-0.02(-0.04$ to 0.00$)$ & $\mathrm{p}=0.29$ \\
& $0.18(-0.22$ to 0.58$)$ & $-11.7(-26.5$ to 3.3$)$ \\
\hline
\end{tabular}

Results are given as regression coefficients with $95 \%$ confidence intervals (in parentheses) and $\mathrm{p}$ values. $+\mathrm{ve})$ and $11(24.4 \%)$ of the 45 control patients $(\mathrm{Pa}-\mathrm{ve})$ had an underlying cause of bronchiectasis (allergic bronchopulmonary aspergillosis, previous pulmonary tuberculosis, hypogammaglobulinaemia, primary ciliary dyskinesia, Kartagener's syndrome, or Young's syndrome).

$P$ aeruginosa infection was associated with increasing age $(\mathrm{p}<0.01)$ and a lower $\mathrm{FEV}_{1}$ $(\mathrm{p}<0.02$; table 1$)$. On logistic regression infection by $P$ aeruginosa was independently associated with a longer duration of sputum production (odds ratio $1.06 ; 95 \%$ CI 1.01 to $1.12 ; \mathrm{p}=0.04)$ but not with increasing age at onset of sputum production $(p=0.08)$, pack years of smoking $(p=0.41)$, sex $(p=0.72)$, a history of asthma $(p=0.33)$, or the presence of an underlying cause of bronchiectasis $(\mathrm{p}=$ $0.97)$.

The extent and severity of bronchiectasis, the severity of bronchial wall thickening, and the extent of decreased attenuation were strikingly greater in the $\mathrm{Pa}+$ ve group than in the $\mathrm{Pa}$ - ve group (fig 1 ; table 2). Both observers agreed that cystic and/or varicose bronchiectasis was present in 15 of the 67 patients $(22.5 \%)$; the kappa coefficient of agreement was 0.71 . Cystic and/or varicose bronchiectasis was present more often in the $\mathrm{Pa}+$ ve group $(10 / 22,45 \%)$ than in the $\mathrm{Pa}-$ ve group $(5 / 45$, $11 \%$; $\mathrm{p}<0.0002$ ).

Multiple regression analysis was performed to evaluate associations between the presence of $P$ aeruginosa and morphological features on the CT scan independently of other factors associated with $P$ aeruginosa (age and duration of sputum production). As shown in table 3 , the presence of $P$ aeruginosa was associated with more extensive and severe bronchiectasis (both $\mathrm{p}<0.0005)$, greater bronchial wall thickening $(p<0.0005)$, and more extensive decreased attenuation $(\mathrm{p}<0.005)$. A longer history of sputum production was associated with a more extensive decreased attenuation score $(\mathrm{p}<0.05)$ and a shorter history of sputum production was associated with more extensive centrilobular mucus plugging $(\mathrm{p}<0.04)$. There were no independent relationships between morphological features of the CT scan and patient age.

Logistic regression was performed to evaluate independent associations between individual CT morphological features and $P$ aeruginosa infection. The presence of $P$ aeruginosa was independently related to increasing bronchial wall thickness (odds ratio $1.74 ; 95 \%$ CI 1.00 to $1.73 ; \mathrm{p}<0.05$ ) but not to the severity of bronchial wall dilatation, the global extent of bronchiectasis, or the extent of regional air trapping.

\section{Discussion}

We have shown that patients who persistently expectorate sputum infected with $P$ aeruginosa have more extensive and severe bronchiectasis on thin section CT scanning than patients whose sputum is not infected with $P$ aeruginosa. The global decreased attenuation score in these 
patients was also significantly greater and related to the duration of sputum production.

$P$ aeruginosa produces several virulence factors which are thought to result in the disorganisation of epithelial cells, loss of cilia, and mitochondrial damage that has been observed in vitro when intact respiratory mucosa is exposed to this pathogen. ${ }^{450-23} P$ aeruginosa preferentially adheres to mucus and damaged epithelial cells rather than to cilia or unciliated cells. ${ }^{5}$ In patients with bronchiectasis and cystic fibrosis in whom the mucus is poorly cleared $P$ aeruginosa may persist for longer in the airway secretions and produce toxins which stimulate a vicious circle of chronic inflammatory damage to the mucosa, disrupting the normal host defence mechanisms and encouraging persistence of infection. ${ }^{24}$ This hypothesis may explain why in our study patients infected with $P$ aeruginosa had significantly more extensive and severe bronchiectasis, bronchial wall dilatation and thickening with a higher prevalence of cystic and varicose patterns present than non-infected patients, this relationship being preserved even after adjustment for the duration of sputum production.

Various hypotheses have been proposed for the mechanism of airway damage in bronchiectasis. Elastase detected in high levels in the sputum of patients with bronchiectasis ${ }^{25}$ impairs ciliary function and damages bronchial epithelium; ${ }^{4}$ its presence in sputum has been shown to be associated with gas trapping. ${ }^{25} P$ aeruginosa produces its own elastase ${ }^{42627}$ and this may further exacerbate airway damage. Areas of decreased attenuation attributable to obliteration of the small airways are commonly observed in patients with a history of chronic purulent sputum production and bronchiectasis on HRCT scanning, ${ }^{28}$ suggesting that disease of the small airways may play an important part in the pathogenesis of bronchiectasis.

Our study confirms that areas of decreased attenuation are a frequent finding in patients with bronchiectasis and patients infected with $P$ aeruginos $a$ achieved a higher global decreased attenuation score than the non-infected patients, indicating a greater degree of small airways disease. The decreased attenuation score in our study was significantly related to the duration of sputum production, suggesting that this feature may be associated with the process of mucus production as demonstrated by in vitro and in vivo experiments. ${ }^{262729}$ However, as there was also a significant negative relationship between the presence of centrilobular mucus plugging and the duration of sputum production, it is possible that centrilobular mucus plugging is indicative of inflammation early in the course of bronchiectasis which tends to regress as the bronchiectasis becomes more established when damage to the small airways by an obliterative bronchiolitis becomes the more dominant feature.

This study clearly shows that patients with bronchiectasis infected by $P$ aeruginosa have a more extensive and severe spectrum of disease present on HRCT scanning than non-infected patients and the single feature on the CT scan that predicted the likelihood of sputum infection by $P$ aeruginosa was increasing thickness of the bronchial wall. Undoubtedly, many complex mechanisms involving the numerous virulence factors produced by $P$ aeruginosa are involved throughout the disease process resulting in damage to bronchi and bronchioles but, at present, these in vivo mechanisms are incompletely understood; whether $P$ aeruginosa preferentially infects the airways of those patients with more severe bronchiectasis $a b$ initio or whether the greater severity of bronchiectasis detected by HRCT scanning is the end result of infection by this organism has not been answered by this study. A reduction in sputum infection by $P$ aeruginosa has been associated with an improvement in lung function in cystic fibrosis, ${ }^{30}$ but it remains to be determined whether a significant improvement in morbidity can be achieved in patients with other forms of bronchiectasis who are chronically infected by this pathogen (although preliminary results appear encouraging ${ }^{31}$ ).

1 Reynolds HY, Di Sant' Agnese PA, Zierdt CH. Mucoid Pseudomonas aeruginosa. A sign of cystic fibrosis in young adults with chronic pulmonary disease? $\mathcal{F} A M A$ 1976;236: 2190-2.

2 Rivera M, Nicotra MB. Pseudomonas aeruginosa mucoid strain. Its significance in adult chest disease. Am Rev Respi Dis 1982;126:833-6.

3 Valerius NH, Koch C, Hoiby N. Prevention of chronic Pseudomonas aeruginosa colonisation in cystic fibrosis by early treatment. Lancet 1991;338:725-6.

4 Amitani R, Wilson R, Rutman A, Read R, Ward C, Burnett $\mathrm{D}$, et al. Effects of human neutrophil elastase and Pseudomonas aeruginosa proteinases on human respiratory epithelium. Am f Respir Cell Mol Biol 1991;4:26-32.

5 Tsang KWT, Rutman A, Tanaka A, Lund V, Dewar A, Cole PJ, et al. Interaction of Pseudomonas aeruginosa with human PJ, et al. Interaction of Pseudomonas aeruginosa with human
respiratory mucosa in vitro. Eur Respir f 1994;7:1746-53.

6 Cole PJ. Bronchiectasis. In: Brewis RAL, Gibson GJ, Geddes DM, Corrin B, eds. Respiratory medicine. 2nd edn. Geddes DM, Corrin B, eds. Respiratory medicin
London: Balliere Tindall, 1995: 1286-316.

7 Munro NC, Barker A, Rutman A, Taylor G, Watson D, MacDonald-Gibson W, et al. The effect of pyocyanin and 1-hydroxyphenazine on in vivo tracheal mucus velocity. $\mathscr{f}$ Appl Physiol 1989;67:316-23.

8 Lapa e Silva JR, Guerreiro D, Noble B, Poulter LW, Cole PJ. Immunopathology of experimental bronchiectasis. $A m$ f Respir Cell Mol Biol 1989;1:297-304.

9 Grenier P, Cordeau M-P, Beigelman C. High resolution computed tomography of the airways. F Thorac Imagin 1993;8:213-29.

10 Grenier P, Maurice F, Musset D, Menu Y, Nahum H. Bronchiectasis: assessment by thin section CT. Radiology 1986; 161:95-9.

11 Young K, Aspestrand F, Kolbenstvedt A. High resolution $\mathrm{CT}$ and bronchography in the assessment of bronChiectasis. Acta Radiol 1991;32:439-41.

12 Nagaki $M$, Shimura S, Tanno Y, Ishibashi T, Sasaki S, Takishima T. Role of chronic Pseudomonas aeruginosa infection in development of bronchiectasis. Chest 1992;102: 1464-9.

13 Wells AU, Desai S, Whetton C, Wilson R, Cole P. The isolation of Pseudomonas aeruginosa from sputum in idiopathic bronchiectasis: an association with extensive disease and severe airflow limitation. Am Rev Respir Dis 1993 147:A645.

14 Quanjer PH. Standardised lung function testing. Clin Respi Physiol 1983;19(Suppl 15):1-95.

15 Naidich DP, McCauley DI, Khouri NF, Stitik FP, Siegelman SS. Computed tomography of bronchiectasis. $\mathcal{F}$ Comput Assist Tomogr 1982;6:437-44

16 Reiff DB, Wells AU, Carr DH, Cole PJ, Hansell DM. CT findings in bronchiectasis: Limited value in distinguishing between idiopathic and specific types. AfR 1995;165: 261-7.

17 Reid LM. Reduction in bronchial subdivision in bronchiectasis. Thorax 1950;5:233-47.

18 Muller NL, Miller RR. Diseases of the bronchioles: CT and histopathologic findings. Radiology 1995;196:3-12.

19 Brennan P, Silman A. Statistical methods for assessing observer variability in clinical measures. BMf 1992;304: 1491-4.

20 Pitt TL. Biology of Pseudomonas aeruginosa in relation to pulmonary infection in cystic fibrosis. F R Soc Med 1986; 79:13-18

21 Wilson R, Pitt T, Taylor G, Watson D, Macdermot J, Sykes $\mathrm{D}$, et al. Pyocyanin and 1-hydroxyphenazine produced by Pseudomonas aeruginosa inhibit the beating of human respiratory cilia in vitro. $\mathcal{F}$ Clin Invest 1987;79:221-9. 
22 Read RC, Roberts P, Munro N, et al. Effects of Pseudomonas aeruginosa rhamnolipids on mucociliary transport and mucociliary beating. I Appl Physiol 1992;72:2271-7.

23 Ramphal R, Pyle M. Further characterisation of tracheal receptor for Pseudomonas aeruginosa. Eur 7 Clin Microbiol 1985;4:160-2.

24 Cole PJ, Wilson R. Host microbial interrelationships in respiratory infection. Chest 1989;95:217-21S.

25 Stockley RA, Hill SL, Morrison HM, Starkie SM. Elastolytic activity of sputum and its relation to purulence and to
lung function in patients with bronchiectasis. Thorax 1984 ; lung function

26 Somerville M, Rutman A, Wilson R, Cole PJ, Richardson PS. Stimulation of secretion into human and feline airways by Pseudomonas aeruginosa protease. F Appl Physiol 1991; 70:2259-67.

27 Somerville M, Taylor GW, Watson D, Rendell NB, Rutman A, Todd $\mathrm{H}$, et al. Release of mucus glycoconjugates by
Pseudomonas aeruginosa rhamnolipids into feline trachea in vivo and human bronchus in vitro. Am $\mathcal{F}$ Respir Cell Mol Biol 1992;6:116-22.

28 Hansell DM, Wells AU, Rubens MB, Cole PJ. Bronchiectasis: functional significance of areas of decreased attenuation on expiratory CT. Radiology 1994;193:36974.

29 Klinger JD, Tandler B, Liedtke CM, Boat TF. Proteinase of Pseudomonas aeruginosa evoke mucin release by tracheal epithelium. F Clin Invest 1984;74:1669-78.

30 Warren ER, Elliot GR, Warwick WJ, Clawson CC. Reduction of sputum Pseudomonas aeruginosa density by antibiotics improves lung function in cystic fibrosis more than do bronchodilators and chest physiotherapy alone. $A m$ Rev Respir Dis 1990;141:914-21.

31 Rayner CFJ, Tillotson G, Cole PJ, Wilson R. Efficacy and safety of long-term ciprofloxacin in the management of severe bronchiectasis. F Antimicrob Chemother 1994:34: $149-56$. 